\title{
Chemical State Mapping via Soft X-rays using a Wavelength Dispersive Soft X-ray Emission Spectrometer with High Energy Resolution
}

H. Takahashi ${ }^{1}$, N. Handa ${ }^{1}$, T. Murano ${ }^{1}$, M. Terauchi ${ }^{2}$, M. Koike ${ }^{3}$, T. Kawachi ${ }^{3}$, T. $_{4}$ Imazono $^{3}$, N. Hasegawa $^{3}$, M. Koeda ${ }^{4}$, T. Nagano ${ }^{4}$, H. Sasai ${ }^{4}$, Y. Oue $^{4}$, Z. Yonezawa ${ }^{4}$ and S. Kuramoto ${ }^{4}$

1 EO Component BU, JEOL Ltd. ; 1-2 Musashino, 3-chome, Akishima, Tokyo 196-8558, Japan.

2 Institute for Multidisciplinary Research for Advanced Materials, Tohoku University ; 1 -1 Katahira, 2chome, Sendai 980-8577, Japan.

3 Quantum Beam Science Directorate ; Japan Atomic Energy Agency; 1-7 Umemidai, 8-chome, Kizugawa 619-0215, Japan.

4 Device Department, Shimadzu Corp.; 1 Nishinokyo-Kuwabaracho, Nakagyo-ku, Kyoto 604-8511, Japan.

A new wavelength dispersive soft X-ray emission spectrometer (WD-SXES) consisting of X-ray reflection mirrors, newly developed diffraction gratings, and a detector of a charged-couple device (CCD) has been developed for soft X-ray emission spectroscopy $[1,2,3]$. The WD-SXES with two types of diffraction gratings, JS50XL and JS200N, nominally covering an energy range between 50 and $210 \mathrm{eV}$ and with a high sensitive back-thin-type CCD as the detector has successfully been installed to electron probe X-ray microanalyzers (EPMAs) for commercial use [4]. The energy resolution of this WD-SXES is nominally $0.3 \mathrm{eV}$, which is one order of magnitude better than that of conventional WDSs with layered dispersion elements used for detecting light elements in EPMAs. One other useful characteristic of this WD-SXES is parallel detection of the X-rays, which makes possible to operate just like conventional energy dispersive spectrometers for EPMAs. In this presentation, chemical state mapping is reported by means of soft X-rays using this high energy resolution WDSXES.

In this study a JXA-8100 EPMA with the WD-SXES was used for chemical state mapping of an Al-B alloy as shown in Fig. 1. A backscattered electron image of the alloy is shown in Fig. 2. It mainly consists of two phases; one with brighter and the other with darker contrast. The former is aluminum matrix and the latter is $\mathrm{Al}_{2} \mathrm{~B}$. To acquire the emission spectral mapping the following conditions were used: the accelerating voltage of $7 \mathrm{kV}$; the beam size of about $1 \mu \mathrm{m}$; the beam current of $0.1 \mu \mathrm{A}$; the $\mathrm{X}$ ray emission spectral range of 55 and $215 \mathrm{eV}$ dispersed by JS200N grating; the pixel number of specimen of $120 \times 120$ pixels; the scan step interval in the stage movement mode of $0.5 \mu \mathrm{m}$; and the acquiring time per pixel of $30 \mathrm{~s}$. Four peaks corresponding to Al-L, B-K ( $\mathrm{n}=1$ and 2$)$ and C-K $(n=2)$ are clearly observed with high energy resolution. Here, $\mathrm{n}$ denotes the spectral order. Two spectra extracted from the $3 \times 3$ pixels of aluminum matrix and of $\mathrm{Al}_{2} \mathrm{~B}$ inclusion are shown in the energy range between 55 and $85 \mathrm{eV}$ in Fig. 4. In each spectrum the Al-L emission is observed with a sharp edge in the higher energy side. The edge of the aluminum matrix corresponds to the Fermi energy of aluminum metal. It should be noted that the corresponding edge of $\mathrm{Al}_{2} \mathrm{~B}$ is shifted to higher energy side by about $1 \mathrm{eV}$. Using the acquired and stored spectrum data, chemical state mapping was performed using a reconstruction program. The energy range of ROI-1 (region of interest) was selected from 72 to $73.5 \mathrm{eV}$ whereas that of ROI-2 was from 73.5 to $75 \mathrm{eV}$ as shown in Fig. 4. In the former range is included the Al-L emission spectrum peak of metallic aluminum excluding that of $\mathrm{Al}_{2} \mathrm{~B}$, whereas in the latter the situation is the other way round. The resulting maps are shown in Fig. 5. The contrast in the ROI-1 map is reversed in the ROI-2 map as expected. Note that the difference between two ROIs is only $1.5 \mathrm{eV}$. The study confirms that the high energy resolution performance of the WDSXES system is useful to map out difference in the chemical state. In addition, other characteristic soft 
X-ray lines such as Li-K (54 eV), Be-K (109 eV), B-K (183 eV), Mg-L (50 eV), Al-L (73 eV), Si-L $(91 \mathrm{eV})$, P-L $(119 \mathrm{eV})$, S-L $(149 \mathrm{eV})$ and Cl-L $(184 \mathrm{eV})$ are located in the energy range covered with the present WD-SXES system. The detectable energy range can be extended to the K emission spectra of $\mathrm{C}, \mathrm{N}, \mathrm{O}$ and $\mathrm{F}$ for the use of higher order X-ray spectra. The WD-SXES will certainly be used in the wide field for the characterization of various materials, especially of advanced materials.

References

1. M.Terauchi, M.Koike, K.Fukushima and J.Kimura: 16th Intl. Conf. Microscopy , 892 (2006).

2. M.Terauchi, et al., J. Electron Microscopy, 61, 1 (2012).

3. T.Imazono, et al., Appl. Opt. 51, 2351 (2012).

4. H.Takahashi, et al., Microscopy and Microanalysis, 16 (supple. 2), 34 (2010).

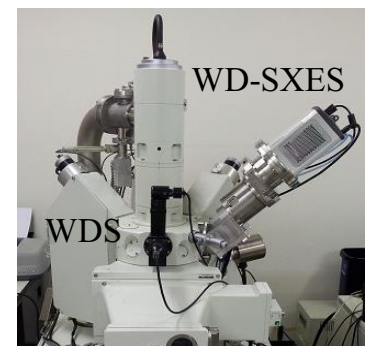

Fig.1 Newly developed WD-SXES installed to an EPMA of JEOL JXA-8100

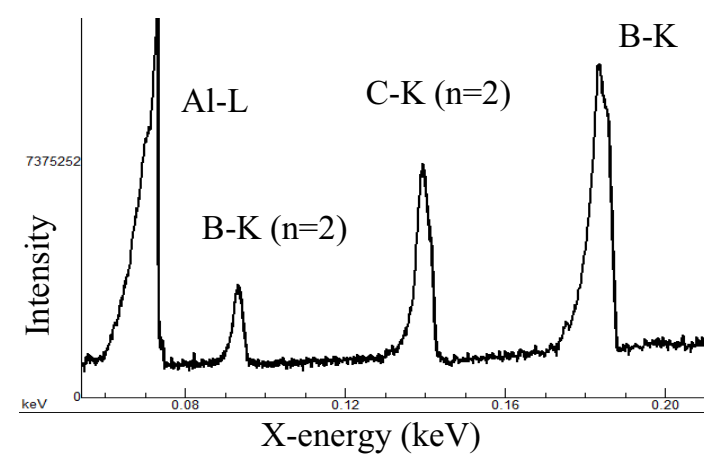

Fig. 3 Cumulative spectrum from all the pixels by spectrum map

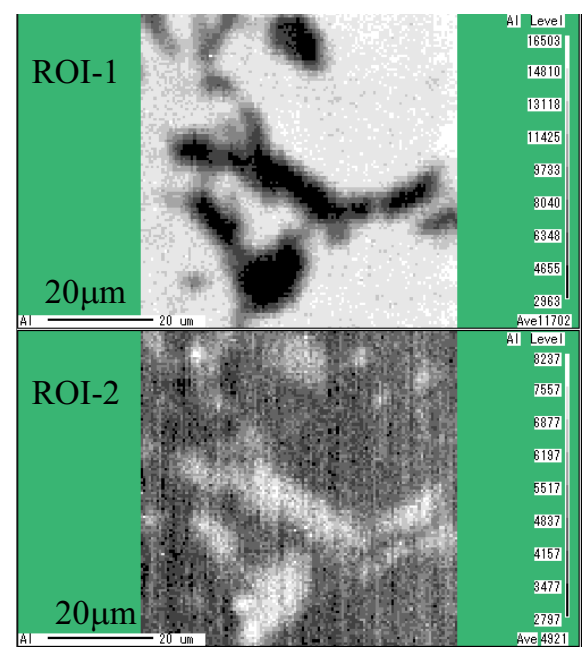

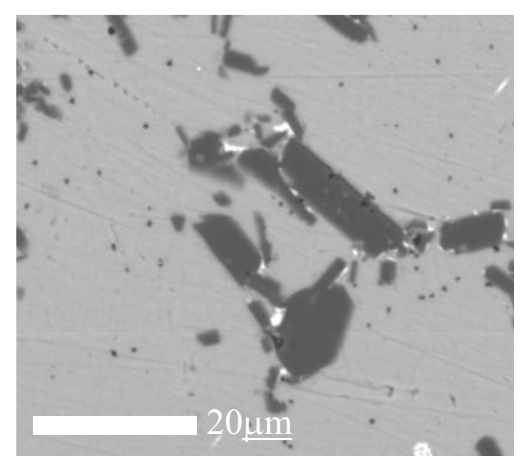

Fig. 2 A backscattered electron image of Al-B alloy

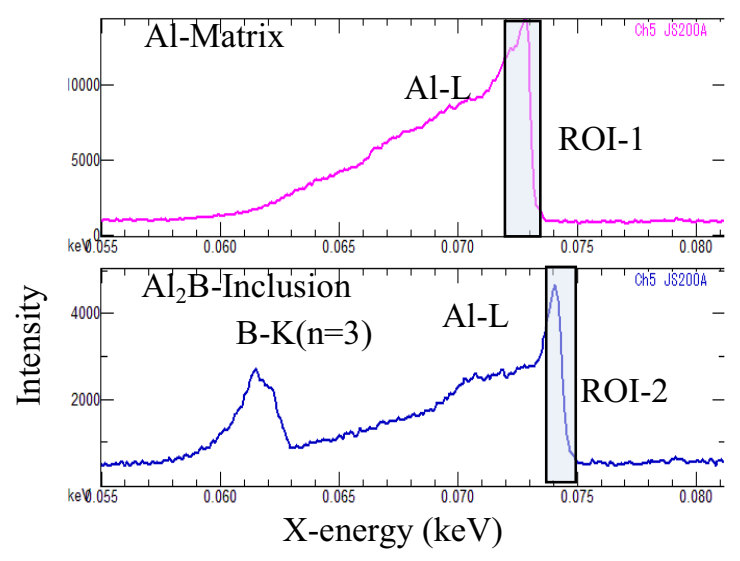

Fig. 4 Comparison of Al-L emission spectra between $\mathrm{Al}$ matrix and $\mathrm{Al}_{2} \mathrm{~B}$ inclusion in Al-B alloy. ROI-1 (72-73.5 eV) and ROI-2 (73.5- $75 \mathrm{eV}$ ) were selected.

Fig. 5 Comparison of Al-L chemical state maps between $\mathrm{Al}$ matrix (ROI-1; 72-73.5 eV) and $\mathrm{Al}_{2} \mathrm{~B}$ inclusion (ROI-2; and $\mathrm{Al}$ matrix $73.5-75 \mathrm{eV}$ ). 